\title{
Force feedback textual and graphic displays for the blind
}

\section{Wooseob Jeong}

School of Information Studies University of Wisconsin - Milwaukee Bolton Hall

5th Floor 3210 N. Maryland Avenue Milwaukee, WI 53202

wjj8612@uwm.edu

Using the force feedback technology, which has been used in video games for years, prototypes of touchable online Braille generator and touchable online graphical display have been developed for visually impaired or blind users. Without expensive devices, these prototypes let blind people access information on the web by touching the output Braille displays or by scrolling over tactile graphical displays with a force feedback mouse. User studies have been conducted with visually impaired people and the data collected provide valuable information about the optimal conditions for these prototype force feedback displays, such as how strong the force should be and how big the Braille dots should appear. The end product of this research will enable visually impaired people to enjoy information on the web, including images, more freely.

Introduction

In the United States, issues concerning information access for those with disabilities have been addressed nationally. Section 508 of the Rehabilitation Act requires federal agencies to make their electronic information accessible to people with disabilities, mainly those who are visually impaired (http://www.section508.gov/ ). Also, the Library of Congress launched a Web-Braille service for the blind in 1998, which continues today (http://www.loc.gov/nls/). With the upsurge of information stored on the Internet, the importance of these issues cannot be overemphasized.

Many products have been developed to help the visually impaired use technology. Several Braille output (pad) and input devices are available such as the Braille Notetaker (http://www.artictech.com/ ). Voice synthesizers for screen readers like JAWS (http://www.freedomscientific.com/fs_products/software_jaws.asp), are also available. 
While these products are mainly for textual information, recent developments put more focus on graphical displays. The American National Institute of Standards and Technology proposed a 'Pins' Down Imaging System for the Blind (http://www.nist.gov/public_affairs/factsheet/visualdisplay.htm ) and Uniplan Ltd. in Japan and KSG America LLC (http://www.kgs-america.com/dvs.htm ) also produced other products based on similar ideas. Software like the Duxbury Braille Translator (http://www.duxburysystems.com/ ) can translate plain text into Braille output which can then be used for embossed printing. However, such products are fairly expensive, ranging from hundreds to several thousands of dollars, in addition to the cost of computers.

Fortunately, there is a potentially promising solution. Based on the technology used in prior research (Jeong \& Gluck, 2002), I could make an online Braille generator which can either be touched or felt by a force feedback mouse similar to the type used in some video games (Jeong, 2005a).

This application has several advantages over the existing devices. First of all, it does not require any expensive special devices, only a $\$ 20$ mouse which is readily available. Also, the technology is available anytime, anywhere as long as there is access to the Internet. Another advantage is that this technology utilizes the existing Braille skills of visually impaired people. Furthermore, the same technology can be used for image displays. We can create a virtual museum for the blind where they can touch objects that are displayed alongside the Braille description of them.

\section{Literature Review}


Several studies already attempted to apply force feedback technology to assist blind people's computing. Ramstein (1996) conducted a pilot study to apply haptics to Braille. Yu and Brewster (2002) compared multimodal virtual reality versus printed medium in visualization for blind people in terms of force feedback. Tzovaras and others (2002) tried to implement virtual reality interface with force feedback for the blind people. Ramloll and others (2000) attempted haptic line graphs with sound for the blind student.

Emery and others (2003) tested multimodal interface including haptic with 29 older adults to find the all participants performed well under auditory-haptic bimodal feedback. Jacko and others (2003) tested multimodal interface with 29 normal vision older adults and 30 visually impaired older adults to find that for some cases, non-visual (auditory or haptic) feedback forms demonstrated significant performance gains over the visual feedback form. Jeong and others (2004) proposed the interactive system which combines an immersive virtual environment with human-scale haptic interface.

\section{Purpose of Study}

The purpose of this study was to explore the feasibility to use force feedback technology already used in video games to facilitate blind people's access to both text and graphic information on the web. Both quantitative and qualitative data were collected to identify optimal conditions with which the prototypes can serve best for the blind.

\section{Prototype Development}

Force feedback technology has been used for many years in video games and has expanded to other areas such as surgical operations and dangerous mechanical processes. I applied this technology to GIS to solve ambiguous multi-color displays for multi-variable thematic maps (Jeong \& Gluck, 2002; Jeong, 2005b).

The online Braille generator basically translates text on the Web into a Braille display, letting the user feel the dots in Braille with a vibrating mouse. The touchable image generator converts each pixel of an image into a dot which can be felt through force feedback.

For touchable images, a dot-based input form is used to generate outputs with force feedback. With the same mechanism, users felt a tactile effect when they mouse over the force feedback dots (Figure 3). In both cases, a low-priced (\$20) force feedback mouse 
was used (Figure 4).

The prototype interface was developed using Immersion Studio (http://www.immersion.com ), JavaScript, PERL/CGI and ASP (Active Server Pages). Logitech's iFeel mouse is used for force feedback output (Figure 1). There is an input text box which can be filled with any plain text; once it is submitted, the text will be instantly translated in Braille (Figure 2). When the user moves the mouse over each dot on the screen, it vibrates with a given force. While users are exploring the screen with the vibrating mouse, force feedback dots provide a similar tactile effect for the users such as Braille displays on the screen.

In future projects, these manual conversion programs will be upgraded to automatic conversion programs, with which any texts or images on the web can be grabbed by their URLs and converted into touchable format for the blind people.

\section{User Study}

To make these prototypes more usable in reality by visually impaired people, user studies were conducted with 19 totally blind people who can read Braille, in Milwaukee area, the state of Wisconsin, USA. The sample size of 19 may sound less than desirable. However, considering the fact that there are not so many people who are totally blind and can read Braille, the number is far more than those found in other research on blind people.

Participants were recruited through several organizations such as the university's Student Accessibility Center, vision teachers in local school districts, public libraries with centers for the blind and non-profit organizations for physically impaired people. Participants provided valuable information about optimal conditions for the prototypes. This information will lead to force feedback displays that eventually enable visually impaired people to enjoy the vast amount of information on the web without expensive devices. It will provide alternative ways to access the virtually unlimited text and graphical information on the Internet, which is often not accessible by the blind.

\section{Experimental Procedure}

Experiments were conducted either at the organization's offices, or at the participants' homes, depending on the participants' preference. Each session last no more than 60 minutes. Participants were asked to try different interfaces of force feedback Braille or graphic outputs with various dot sizes and magnitudes of force. They used a tactile mouse on a notebook computer, and after exploring every option they were asked to select the 
most comfortable settings for their touch senses: how big (or small) the dots should be, how strong the force should be, what kind of force feedback should be used (vibration or friction), and their general opinions of the prototypes.

Interviews were accompanied by the experiments so that both quantitative and qualitative data could be collected from the experiments. Interviews were transcribed for qualitative data analysis.

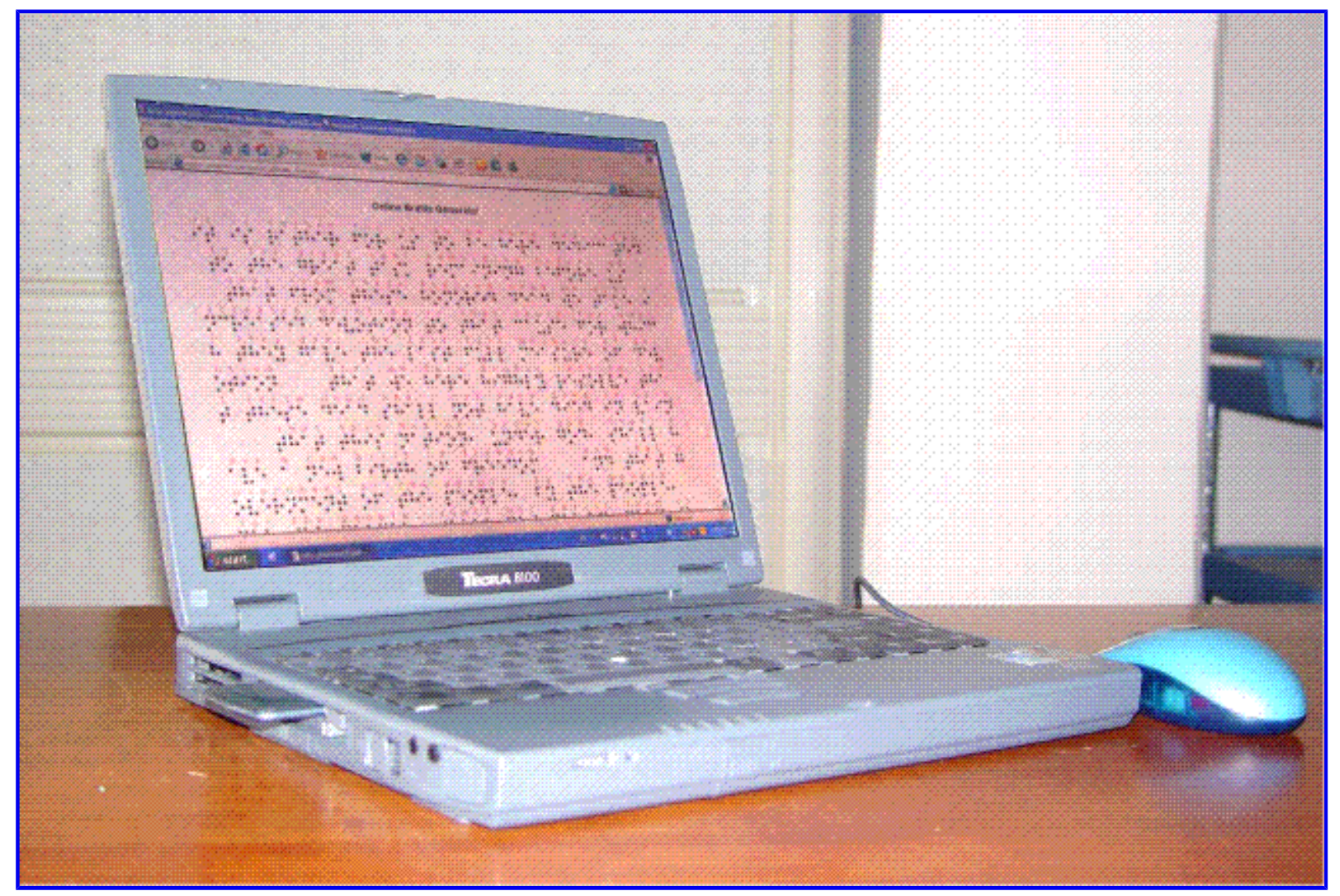

Figure 1. Experimental Setting 


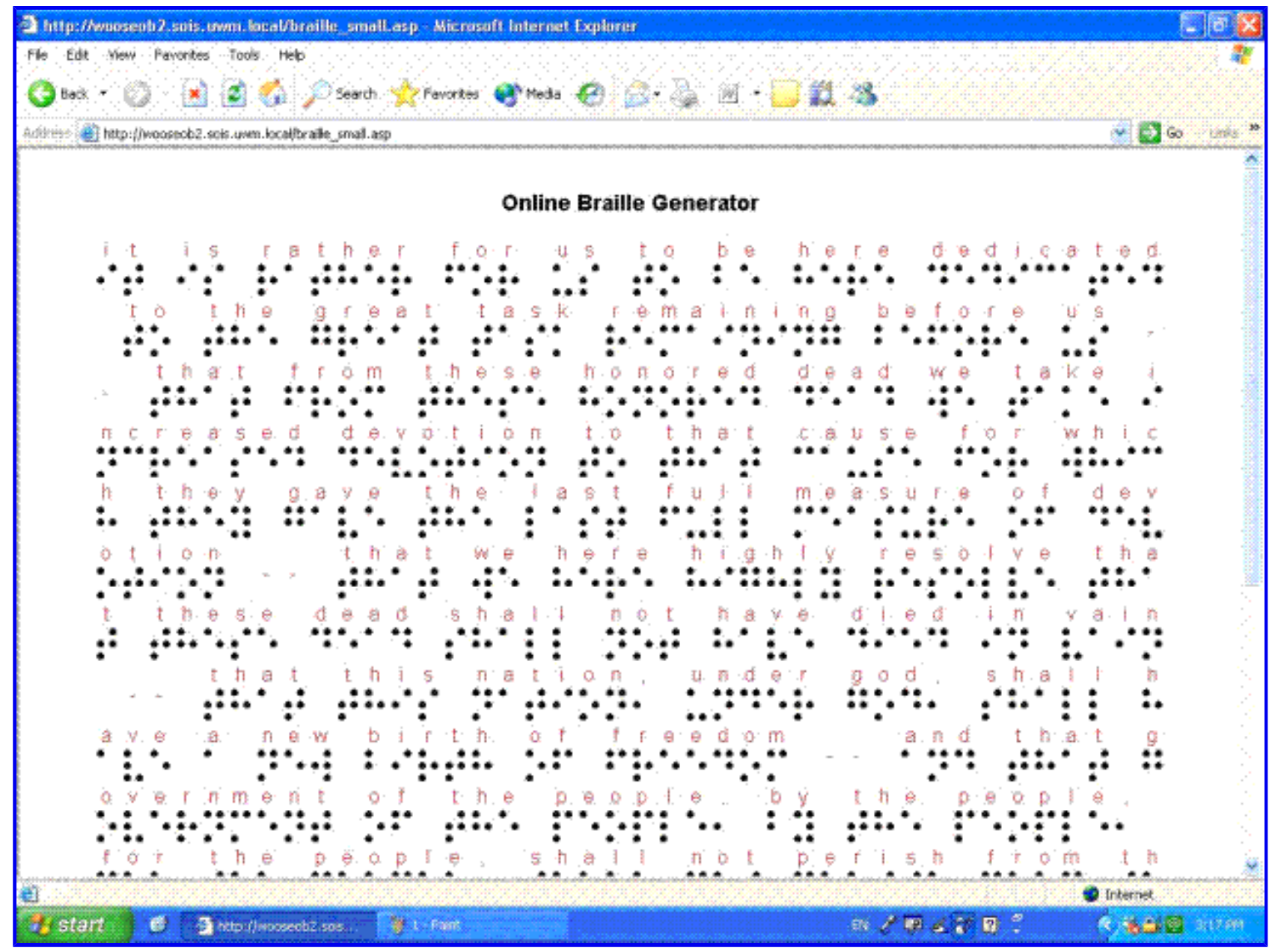

Figure 2. Touchable Braille Screen Output 


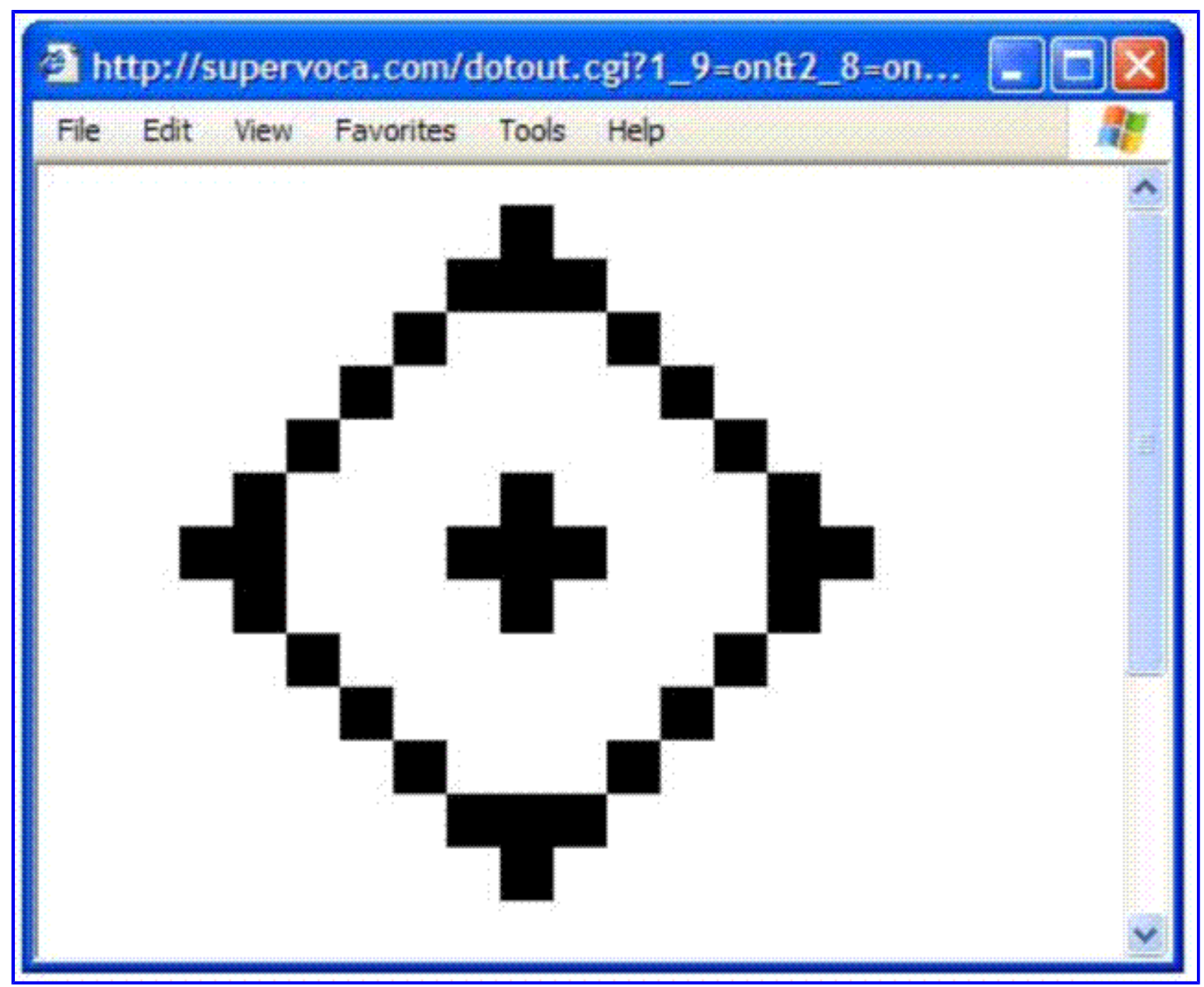

Figure 3. Touchable Image Output 


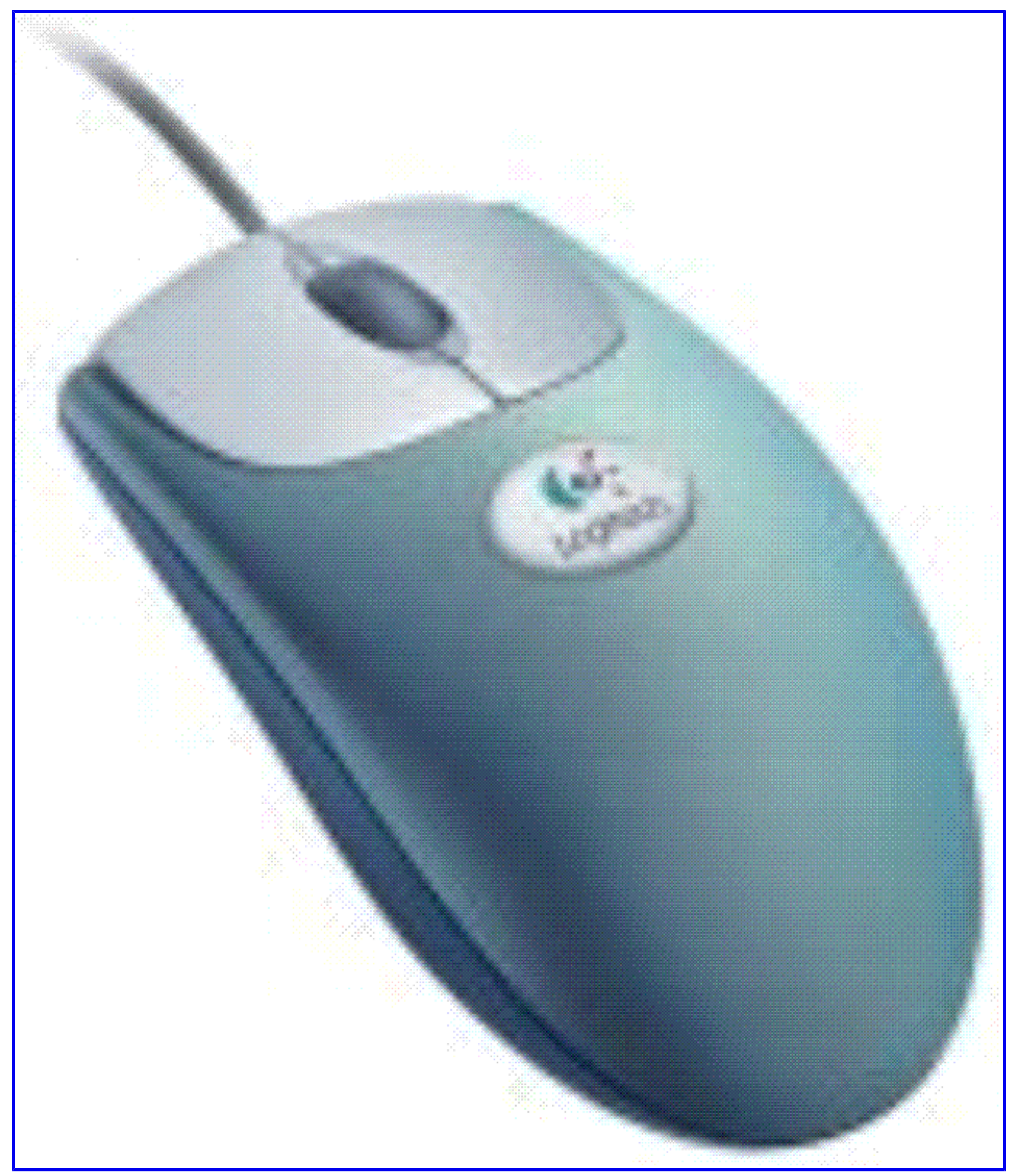

Figure 4. Inexpensive Force Feedback Mouse

\section{Result}

Even though there were only 19 participants, a number of issues were clearly identified. It is encouraging to see that all of the participants could identify Braille characters with force feedback by a mouse with the guidance of the researcher. All the participants agreed that 
this prototype would be useful with reasonable training. This implies a great feasibility of this prototype. They prefer the biggest dot size (30 pixels in diameter) and the possibly strongest force for maximum perception of the force feedback effect.

However, the currently dominant voice synthesizer software makes the prototype less attractive to the blind people. At least two participants clearly mentioned their current Braille pads are enough for them as well. It seems that they are not willing to invest their time and efforts in a new device.

They got more excited about the force feedback graphic displays. That is a natural reaction because there is no practical alternative way for them to feel graphics on computers. There are experimental devices available, but they are either quite expensive or still remaining in laboratories. The blind participants suggested that this graphical prototype could be used for geometry and geography easily and effectively.

\section{Discussion}

\section{Blind People's Navigation by Mouse}

Blind people never use mouse for their computing, so using mouse itself is a big challenge for them. A vision person uses a mouse with a hand and eyes: moving a mouse watching the mouse cursor on the screen. However, for the blind people, without seeing the mouse cursor, it is very difficult to identify the mouse's position. Directions to move and distance between two points are also difficult to grasp. They cannot move the mouse in a straight line since there is no guidance. This problem hinders the effectiveness of the force feedback displays for the blind. However, this issue is not true for only blind people. Some vision people, especially older generation, cannot move mouse easily even with their eyes. One possible solution may be to put some guardrails to help the blind people to differentiate relevant areas from non-relevant ones.

Another issue on mouse is how they hold it. Since they never use mouse, when they are asked to hold a mouse, most blind people really "hold" firmly the mouse, too firm to move it or feel the enough force. So far only one participant could use the mouse successfully, who is a music major college student with 15 years of piano playing experiences. This implies that a significant learning session will be required to let the blind people use the mouse freely.

\section{Different Image Conceptualization}


One really interesting finding is that every participant answered "Triangle", when they are asked to guess a shape of " $L$ " by touching the force feedback mouse. The reason may be explained by the fact that the blind's people's image conceptualization process is different from that of vision people. If they are born-blind, they never see things in their eyes. They only conceptualize things by touch and sound, and many times the number of shapes in their minds is limited. In this particular chase, "L" has no meaning for the blind people, while its Braille counterpart has a real meaning for them. So, a triangle shape is the closes one that they can imagine.

\section{Ignorance or Suppress of Graphical Information Need}

During the course of experiments, it is apparent that the blind people's graphical information needs are limited. Basically they are not so interested in graphical displays on computers or electronic devices. It is considered that their graphical information needs are ignored or suppressed by themselves based on their life-time experiences. Rather they tend to resort to the Braille and more recently voice synthesizers. This finding suggested that numerous researches on graphics for the blind may not have an inappropriate direction. In many studies, they lack of experiments with the enough blind people for their new devices. Studies for the blind should be more based on their real information needs, rather than the researchers' expectation with vision.

\section{More Research Needed with Sound}

Since they are already using sound, particularly voice synthesizers predominantly, more sound applications are suggested for the blind. For example, I saw a 13-year boy got immersed with a computer audio game named Grizzly Gulch, and I think audio games have potentials to help the blind children to learn some skills as in the video games for the vision children. The audio games also provide broader research areas for the future studies.

\section{Conclusion}

Numerous devices have been developed to improve accessibility of blind or visually impaired people to information including on the internet. However, they are quite expensive or limited in flexibility, and mainly for the texts. Still there is no reasonable 
graphic display for those people, except for laboratory level's expensive and bulky pin-based external devices.

Compared to those devices, these new prototypes are using established force feedback technology, which has been used in video games for years, with a minimal cost to existing PCs. They are both texts and graphics as well. Blind children are still using hand-made 3D picture books, and the books are labor intensive and time consuming to make. With these prototypes, that kind of problems will be easily resolved.

The final products derived from this study can be used for many purposes nationally and internationally. All the information on the web, whether texts or images, can be delivered to the visually impaired people without those expensive devices. This touchable Braille lets deaf-blind people, who cannot use screen reader software, enjoy the texts on the web, and will help people to learn Braille, even if it's not so appealing hearing blind people.

In terms of images on the web, the prototype has more exciting and enormous potentials since there is no practically usable method for images yet. For examples, children's books can be delivered easily to the blind children, who will touch their images via the force feedback mouse. Maps on local, state, national, or international interests can be delivered to the blind people as well.

These prototypes will help to add another sense, touch, to already blossoming visual and auditory digital libraries. By force feedback technology, new multimodal digital libraries will be accessible by the world.

\section{References}

Emery, V. K. et al. (2003) Toward achieving universal usability for older adults through multimodal feedback Proceedings of CUU'03, November 10-11, 2003, Vancouver, British Columbia, Canada 46-53

Jacko, J. A. et al. (2003) Older adults and visual impairment: what do exposure times and accuracy tell us about performance gains associated with multimodal feedback? Proceedings of CHI2003, April 5-10, 2003, Ft. Lauderdale, Florida 33-40 Jeong, S., Hashimoto, N., \& Makoto, S. (2004) A novel interaction system with force feedback between real - and virtual human Proceedings of ACE'04, June 3-5, 2004, Singapore 61-66

Jeong, W. \& Gluck, M. (2003) Multimodal Geographic Information Systems: Adding Haptic and Auditory Display Journal of the American Society for Information Science and Technology 54(3): 229-242 
Jeong, W. (2001) Exploratory user study of haptic and auditory display for multimodal geographic information systems CHI'2001: Extended abstracts 73-74

Jeong, W. (2005a) Touchable Online Braille Generator ASSETS 2005 188-189 Jeong, W.(2005b) Multimodal Trivariate Thematic Maps with Auditory and Haptic Display ASIST 2005, Charlotte, North Carolina, October 28 - November 2

Ramloll, R. et al. (2000) Constructing sonified haptic line graphs for the blind student: first steps Proceedings of the fourth international ACM conference on Assistive technologies 17-25

Ramstein, C. (1996) Combining haptic and Braille technologies, design issues and pilot study ASSETS'96 37- 44

Tzovaras, D. et al. (2002) Multimodal technologies: Design and implementation of virtual environments training of the visually impaire Proceedings of the fifth international ACM conference on Assistive technologies 41-48

Yu, W. \& Brewster, S. (2002) Multimodal technologies: Multimodal virtual reality versus printed medium in visualization for blind people Proceedings of the fifth international ACM conference on Assistive technologies 57-64 